\title{
The Occurrence of Ideal Plastic State in CP Titanium Processed by Twist Extrusion
}

By Aleksey Reshetov,* Roman Kulagin, Alexander Korshunov, and Yan Beygelzimer

[*] Dr. A. Reshetov

Advanced Forming Research Centre, University of Strathclyde, 85 Inchinnan Drive, Renfrew PA4 9LJ, United Kingdom

E-mail: aleksey.reshetov@strath.ac.uk

Dr. R. Kulagin

Institute of Nanotechnology (INT), Karlsruhe Institute of Technology (KIT), Eggenstein-

Leopoldshafen 76344, Germany

Dr. A. Korshunov

Russian Federal Nuclear Center VNIIEF, 37 Mira Ave., Sarov 607190, Russia

Prof. Dr. Y. Beygelzimer

Department of High Pressure Physics and Promising Technologies, Donetsk Institute for

Physics and Engineering named after A.A. Galkin of the National Academy of Sciences of Ukraine, Pr. Nauki 46, Kyiv, 03028, Ukraine

This paper deals with the analysis of strength and plastic characteristics of commercially pure $(C P)$ titanium specimens as a function of equivalent plastic strain accumulated during Twist Extrusion (TE) process. It is shown experimentally that multipass TE leads to the saturation of yield stress, reduction in area, elongation to failure and uniform elongation. This fact indicates the occurrence of an ideal plastic state in the processed material. The threshold value of accumulated plastic strain for ideal plastic behavior of CP titanium during TE is defined. The strain state and mechanical properties of CP titanium billets processed by TE are studied in both axial and peripheral areas. An explanation for the hardening central zone of the billet during TE is offered. The non-uniformity in mechanical property distribution is quantitatively estimated in the billet cross-section. An effort is undertaken to compare the metal flow during TE and High Pressure Torsion (HPT) process. The analysis of deformation modes on the billet axis during TE and HPT is carried out. It is theoretically and experimentally shown that the differences in final mechanical properties of CP titanium billets processed by TE and HPT are caused by the difference in symmetry of these processes, as well as the different values of hydrostatic pressure in the deformation zone. 


\section{Introduction}

Severe plastic deformation (SPD) processes use large hydrostatic pressure to accumulate high strain in bulk billets and refine the grain size to submicron scale. ${ }^{[1,2]}$ From the standpoint of grain refinement and mechanical property improvement, high-pressure torsion (HPT) process can be considered as the most effective one. ${ }^{[2]}$ This technique was first proposed by P.W. Bridgman, ${ }^{[3,4]}$ whose seminal work devoted to metal forming under the influence of shear strain and high hydrostatic pressure laid down the foundation for SPD area of research. HPT method realizes simple shear deformation mode and allows to process the material by imposing a high level of hydrostatic pressure that can reach up to $9 \mathrm{GPa}^{[5]}$ These distinctive features of HPT process enable us to refine the initial grain structure to nanoscale level, i.e. obtain an average initial grain size of less than $100 \mathrm{~nm} \cdot{ }^{[6]}$ However, the major limitation of this SPD technique is the small size of the processed samples, which are typically discs of diameter $10-15 \mathrm{~mm}$ and thickness $1 \mathrm{~mm}$. A series of studies have been conducted to overcome this limitation and implement HPT method for bulk workpieces ${ }^{[7,8]}$ as well as apply continuous HPT for processing long billets. ${ }^{[9,10]}$

Twist extrusion (TE) is another SPD technique ${ }^{[11,12,13]}$ with a simple shear deformation scheme close to that implemented in HPT. In this process, a bulk billet is pressed through a die comprising two straight channels which separated by a so-called twist channel. The twist channel can be characterized by its twist angle $\chi$, length $L$ and radius $R$ (see Figure 1). 


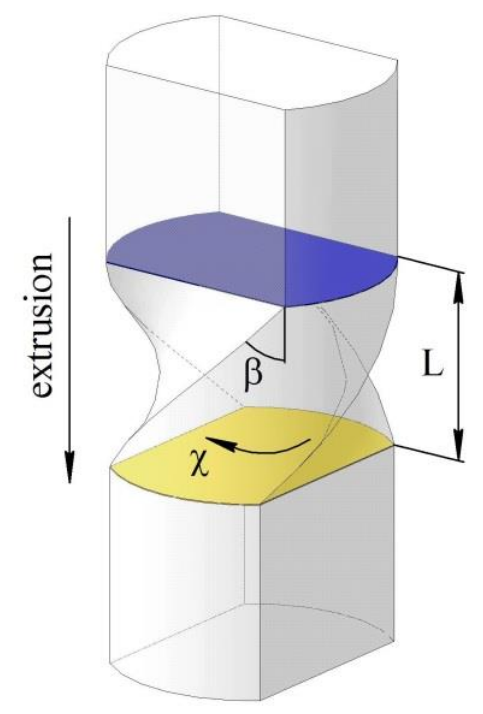

Fig. 1. Twist extrusion scheme: illustration of die angle $\beta$, twist-channel length $L$ and twist angle $\chi$.

A geometrical feature useful for characterizing the twist channel is the angle between the axis and the tangent to the helix of the twist channel. This angle, which will be termed as the die angle $\beta$, relates to other geometrical features of the twist channel as

$\tan \beta=\frac{\chi R}{L}$

TE can be considered, to some extent, as an industrial variant of HPT designed for bulk billet processing. ${ }^{[14]}$ To a first approximation, it can be assumed that TE is equivalent to deforming discrete cross-sections of the billet through HPT. In TE, initially, torsion to a certain angle is achieved in one direction, and then, torsion to the same angle, in the opposite direction, i.e. the deformation is cyclic with the amplitude of the quasi-monotone part equal to half the full strain (see Figure 1). Typically, for the dies used, the accumulated strain per pass is about 1.2. ${ }^{[15]}$ Since the cross-sectional shape of the billet is constant along the extrusion direction, multiple passes of TE can be performed to accumulate large plastic strains in the processed material. 
Owing to the fact that TE is often associated with HPT, some researches assign to TE the same shortcomings as observed for HPT. ${ }^{[2,16]}$ These being significant non-uniformity of deformation, resulting in inhomogeneous distribution of mechanical properties along the sample cross section, as well as pure (or even complete absence of) deformation of the material in the vicinity of the extrusion axis.

The problem of lack of deformation in the center of the sample during HPT was investigated by a number of researchers, who studied grain refinement and accumulated strain around the axis of rotation. ${ }^{[17,18,19]}$ There is some controversy in literature on this subject, as both homogeneous ${ }^{[17,20]}$ and inhomogeneous ${ }^{[18,19,21]}$ distribution of grain size and microhardness in the sample cross-section has been reported. Some researchers confirm that after a large number of HPT revolutions, the material in the cross-sectional center accumulates large plastic strains that results in improvement of mechanical properties and grain refinement to the same degree as observed in the peripheral areas. ${ }^{[20,22,23]}$ Another group of researchers came to the conclusion that processed material located exactly on the axis of rotation stays undeformed even after a large number of HPT revolutions. ${ }^{[18,19,24]}$ For example, A. Vorhauer and R. Pippan carried out a rigorous investigation of the central region of HPT samples from austenitic steel after different number of revolutions. ${ }^{[18]}$ They demonstrated that the center of the samples still has nearly undeformed grains even after a large number of revolutions (up to 16 revolutions). Similar results were reported for other materials as well. ${ }^{[1,24]}$ Y.Z. Tian et al. ${ }^{[19]}$ published experimental data that demonstrated a reduced microhardness in the central regions of $\mathrm{Cu}-\mathrm{Ag}-\mathrm{Zr}$ samples even after 20 revolutions. A. Vorhauer and R. Pippan ${ }^{[18]}$ attributed this discrepancy in the results for the central region to 3 reasons: (1) the difficulty in performing an ideal HPT deformation owing to the unavoidable minor misalignment of the axes of the anvils, (2) the presence of compressive deformation and (3) technical difficulties with detecting small almost undeformed area in the axial zone of the specimen processed by 
HPT. All these reasons make the realization of precise experiments for studying material in the axial zone problematic.

Owing to the similarities between TE and HPT processing schemes and the foregoing considerations, the strain accumulated, microstructure refinement and mechanical properties in the axial zone of a billet processed by TE are of particular interest. A detailed analysis of this topic has not been done so far. This article presents the results of our analysis of TE as a technique for implementation of HPT to bulk samples. The correctness of such a comparison is discussed. The question of deformation in the axial zone of the billet is considered as well. To this end, an analysis of deformation modes on the billet axis during TE and HPT was carried out. Strain state and mechanical properties of commercial purity (CP) titanium billets processed by TE were studied in both axial and peripheral areas of the cross-section. A possible explanation for the hardening of the central zone of the billet during TE is offered. Non-uniformity in the mechanical property distribution is quantitatively estimated in the billet cross-section. The threshold value of accumulated plastic strain for an ideal plastic behavior of CP titanium during TE was defined. The conditions under which the strength and plastic characteristics of $\mathrm{CP}$ titanium attain their saturation values were formulated.

\section{Experimental procedures}

\subsection{TE and mechanical tests}

Hot-rolled CP titanium grade VT1-0, containing $\mathrm{O}-0.12 \mathrm{wt} \% \mathrm{H}-0.01 \mathrm{wt} \% \mathrm{~N}-0.04 \mathrm{wt} \% \mathrm{C}-$ $0.07 \mathrm{wt} \% \mathrm{Fe}-0.18 \mathrm{wt} \%$, was processed by TE. The titanium billets with dimensions shown in Figure 2a were processed by 2 (billet\#1) and 4 (billet\#2) TE passes through a counterclockwise twisted die with a die angle $\beta=60^{\circ}$. TE die channel scheme and its dimensions are shown in Figure $2 \mathrm{~b}$. TE process was performed at a temperature of $350^{\circ} \mathrm{C}$ and $200 \mathrm{MPa}$ backpressure. The average value of plastic strain accumulated in the cross-section of the processed billet amounted to $e \sim 2.4$ and $e \sim 4.8$ after 2 and 4 TE passes, respectively. 


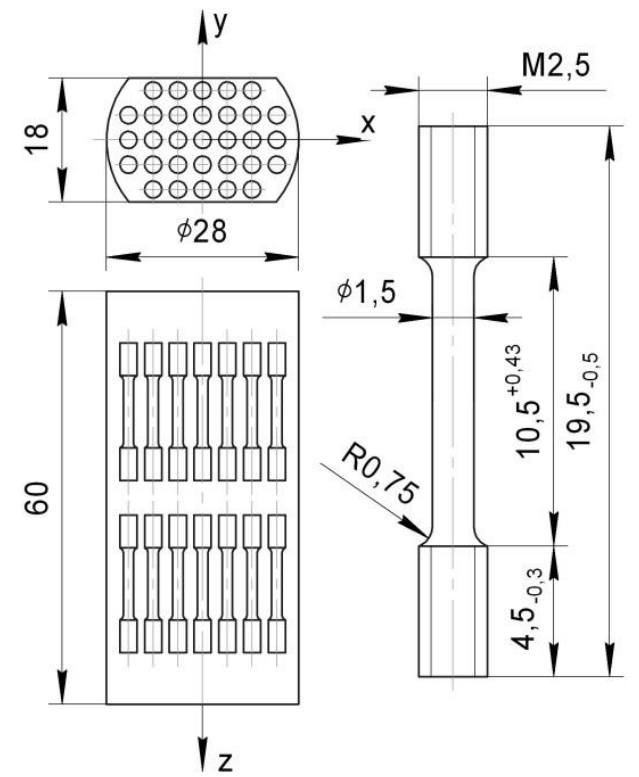

$a$
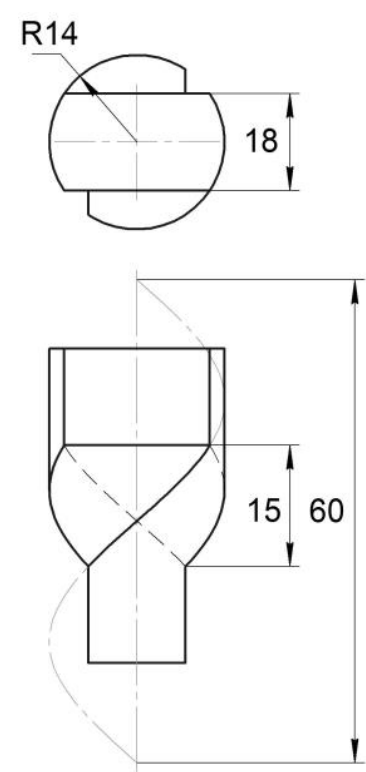

$b$

Fig. 2. (a) Dimensions of the billet, billet cutting scheme and map of tensile specimens; (b) Scheme of the twist die channel used for processing titanium billets.

In order to evaluate the mechanical properties and their homogeneity in the billets crosssections, the processed samples were cut into half along the transverse direction and thereafter these pieces were cut into small specimens along the extrusion axes as it shown in Figure 2a. In such a way, each half of the billet was cut in the direction parallel to the extrusion axis into 31 small tensile test specimens. The specimens had an overall length of $19.5 \mathrm{~mm}$, gage length of $7.5 \mathrm{~mm}$ and a nominal diameter of $1.5 \mathrm{~mm}$ (see Figure 2a). Thus, for each of 31 reference points in the cross-section, two specimens (one from each half of the billet) were tested in tension, in the same manner as it was done in earlier studies. ${ }^{[25,26]}$ The mechanical properties for the reference points were calculated as the arithmetic mean of the results from the two specimens. The coefficient of variation $C V$ was used for quantitative assessment of mechanical properties variability in the cross-section of as-received and processed billets. $C V$ was calculated as the ratio of the standard deviation $S D$ to the mean (average) value of mechanical characteristic in the ross-section $\bar{x}$ : 
$C V=\frac{S D}{\bar{x}} \times 100 \%$

The tests were conducted on a Model 1185 INSTRON testing machine at room temperature and a strain rate of $\sim 1.4 \times 10^{-3} \mathrm{~s}^{-1}$. The strength properties $\sigma_{U T S}$ (ultimate tensile strength) and $\sigma_{Y S}$ (yield stress), as well as plastic characteristics $\delta$ (elongation to failure) and $\psi$ (reduction in area) were measured for all 31 points of each billet cross-section.

\subsection{Finite element analysis procedure}

A large number of publications on TE revealed that the strain state during the process is substantially non-uniform throughout the sample cross-section. ${ }^{[15,27]}$ The value of equivalent plastic strain increases towards the peripheral regions of cross-section, being the smallest near the extrusion axis. Therefore, each sample cut out from a particular region of the billet crosssection after TE processing has a corresponding value of accumulated plastic strain. Thus, the strain values for workpieces cut out from the central and peripheral areas can differ from each other by more than twofold. Therefore, finite element (FE) simulations were performed for an accurate analysis of the strain state in the billet cross-section and for defining accumulated plastic strain for each small-size tensile test specimen. The data obtained from finite element analysis (FEA) and tensile tests were used for plotting true stress - true plastic strain curves describing the strain-hardening behavior of CP titanium processed by TE.

All FE simulations of the TE process were performed using DEFORM 3D ${ }^{\mathrm{TM}}$ Ver.11.0.2 FE package. The tool and die channel geometry, as well as sample dimensions used for the FE model were identical to those described in the previous subsection (see Figure 2). The plunger and TE die were modeled as rigid bodies, and 50,000 deformable tetrahedral elements were used to describe the plastic flow of the billet. Adaptive mesh refinement and automatic 
remeshing were employed to simulate accumulation of large strains during TE process. The velocity of the main plunger was maintained at $3 \mathrm{~mm} \mathrm{~s}^{-1}$ in all simulations.

Von Mises plastic model and the stress-strain relation in the form of Hollomon-type function obtained by fitting compression test results from an earlier study were employed (see ref. ${ }^{[28]}$ for the details):

$\sigma=330 \cdot \varepsilon^{0.19}$

where $\varepsilon$ is von Mises strain and $\sigma$ is true stress of processed material.

A back-plunger with an applied force played the role of back pressure. Taking into account the fact that the value of back pressure during TE is comparable to the yield stress of the processed material, the friction stress reaches high levels and can be defined according to Siebel friction law:

$\tau=m \frac{\sigma_{Y}}{\sqrt{3}}$

where $m=0.05$ is the friction coefficient between titanium and steel. ${ }^{[28]}$

One pass of TE was simulated with the FE model and the equivalent plastic strains defined for each small-size tensile test specimen. To find out the value of accumulated plastic strain for each sample after multiple TE passes, the equivalent strain after the first pass was multiplied by the number of passes.

\section{Results}

3.1. Comparative analysis of deformation mode during TE and HPT processes 
To understand the difference between the scheme of HPT and TE, the velocity gradient tensor was analyzed for both processes. According to Figure 2a, the line of die symmetry is the extrusion axis, which corresponds to the $Z$ axis in the Cartesian frame $(x, y, z)$. Supposing $V$ to be the velocity field of the metal flow in TE, an analysis of the velocity gradient tensor gradV on the $Z$ axis can be carried out. It is evident that $V_{x}=0$ and $V_{y}=0$ on the axis of symmetry. It follows therefrom that on this axis,

$\frac{\partial V_{x}}{\partial z} \equiv 0, \frac{\partial V_{y}}{\partial z} \equiv 0$

For the case of a stretched profile with two axes of symmetry, e.g. rectangular-type profile, the TE die has axis of twofold symmetry. This means that the rotation of the die by $\pi$ radians about the $Z$ axis will not change the components of $\operatorname{grad} V$ tensor on the axis of symmetry. At the same time, such a rotation operation leads to the following transformation of coordinates:

$x^{\prime}=-x, \quad y^{\prime}=-y, \quad z^{\prime}=z$

Substituting (6) into (5) gives:

$$
\frac{\partial V_{z}}{\partial x}=-\frac{\partial V_{z}}{\partial x}, \quad \frac{\partial V_{z}}{\partial y}=-\frac{\partial V_{z}}{\partial y}
$$

It follows from (7) that on the axis of symmetry,

$$
\frac{\partial V_{z}}{\partial x}=0, \frac{\partial V_{z}}{\partial y}=0
$$


Thus, a velocity gradient tensor on the axis of symmetry in TE can be written as follows:

$$
\operatorname{grad} \mathbf{V}=\left[\begin{array}{ccc}
\frac{\partial V_{x}}{\partial x} & \frac{\partial V_{x}}{\partial y} & 0 \\
\frac{\partial V_{y}}{\partial x} & \frac{\partial V_{y}}{\partial y} & 0 \\
0 & 0 & \frac{\partial V_{z}}{\partial z}
\end{array}\right]
$$

As a comparison, let us consider the velocity gradient tensor on the symmetry axis of rotation during HPT process. Under the condition that compressive deformation is absent, $V_{z}=0$ and

$$
\frac{\partial V_{z}}{\partial z}=0
$$

Owing to the fact that during HPT a cylindrical symmetry occurs, on the axis of symmetry

$$
\frac{\partial V_{x}}{\partial x}=\frac{\partial V_{y}}{\partial y}
$$

It follows from the incompressibility condition expressed with (10) that

$$
\frac{\partial V_{x}}{\partial x}=\frac{\partial V_{y}}{\partial y}=0
$$

In addition, due to cylindrical symmetry in HPT, we obtain

$$
\frac{\partial V_{x}}{\partial y}=-\frac{\partial V_{y}}{\partial x}
$$


Thus, the velocity gradient tensor on the axis of symmetry in HPT can be expressed as

$$
\operatorname{grad} \mathrm{V}=\left[\begin{array}{ccc}
0 & \frac{\partial V_{x}}{\partial y} & 0 \\
-\frac{\partial V_{x}}{\partial y} & 0 & 0 \\
0 & 0 & 0
\end{array}\right]
$$

It can be concluded from a comparison of (9) and (14) that the differences in the profile symmetry in TE and HPT lead to differences in the gradV tensor on the axis of symmetry. As a result, the following components of strain rate tensor appear at the center of TE profile:

$$
\dot{e}_{x x}=\frac{\partial V_{x}}{\partial x}, \dot{e}_{y y}=\frac{\partial V_{y}}{\partial y}, \dot{e}_{z z}=\frac{\partial V_{z}}{\partial z}, \dot{e}_{x y}=\frac{1}{2}\left(\frac{\partial V_{x}}{\partial y}+\frac{\partial V_{y}}{\partial x}\right)
$$

These particular components of strain rate tensor are responsible for accumulating large plastic strains and grain refinement in the axial zone during TE. On the other hand, in the case of HPT, all components of strain rate tensor on the symmetry axis are equal to zero. This fact was experimentally proved by Vorhauer and Pippan in ref. ${ }^{[18]}$

Besides the purely geometric considerations listed above, the real TE process has a number of other factors (see detailed description in ref. ${ }^{[27,29]}$ ) that lead to in-plane and out-of-plane distortion of the sample layers (see Figure 3). Among these factors are friction effects, strain hardening behavior of the material, and higher contact pressure in two diagonal corners of the profile. The influence of these factors is studied in ref., ${ }^{[27]}$ where authors have shown that strain at the center of the sample cross section increases with an increase in friction, strain hardening and die angle $\beta$. 


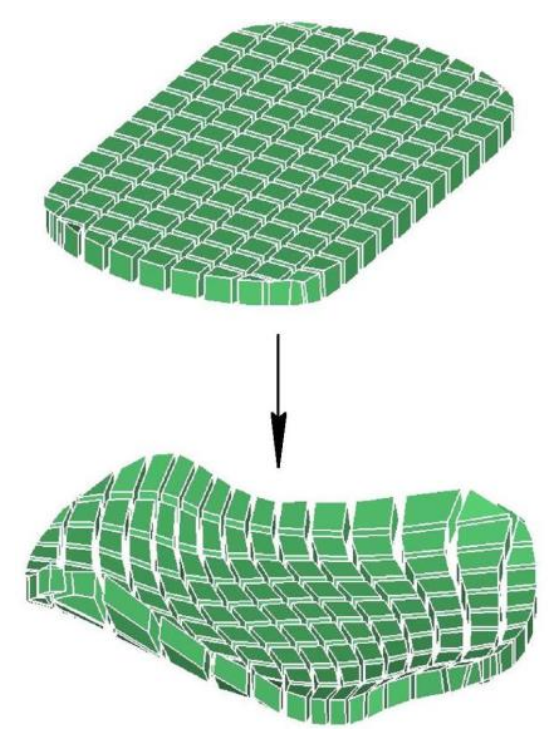

Fig. 3. Illustration of out-of-plane distortion: FE simulation of the workpiece layer during TE.

Figure 4 illustrates the FE simulation results for one pass of TE, which confirms the presence of plastic strain in the axial zone. According to the results, the equivalent plastic strain radially increases from the values of $0.3-0.5$ in the paraxial zone to $2.0-2.5$ in the periphery of the cross-section (see Figure 4). As was shown in an earlier study, ${ }^{[28]}$ the value of strain accumulated at the end of multipass TE processing can be obtained to a first approximation by multiplying the strain value after one pass by the number of TE passes. This means that multipass TE processing can result in large plastic strains accumulated at the center of the profile. The experimental results given below prove significant strain-hardening of the axial zone.

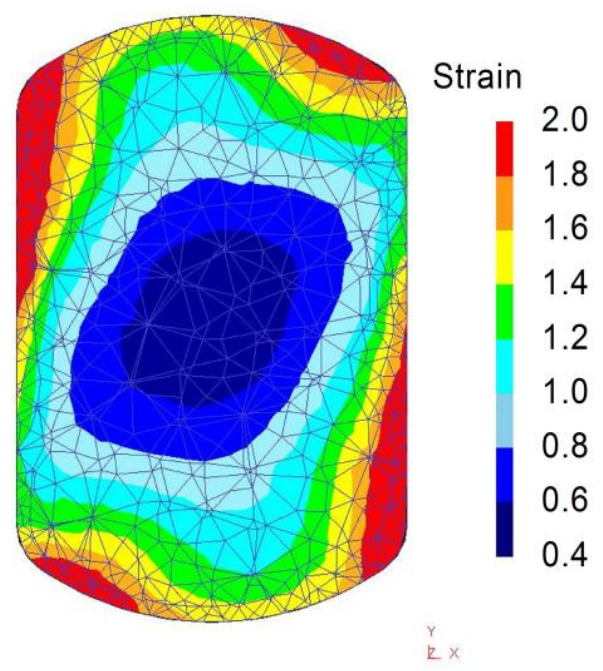


Fig. 4. FE simulation: map of plastic strain values in the cross-section of CP titanium billet processed by 1 TE cycle.

\subsection{Distribution of mechanical properties after 2 and 4 passes of TE}

The results of tensile tests performed on the specimens are summarized in Figure 5, which shows the strength property distribution over the billet cross-section after 2 and 4 TE passes (henceforth referred to as just 2 and 4 passes for brevity).
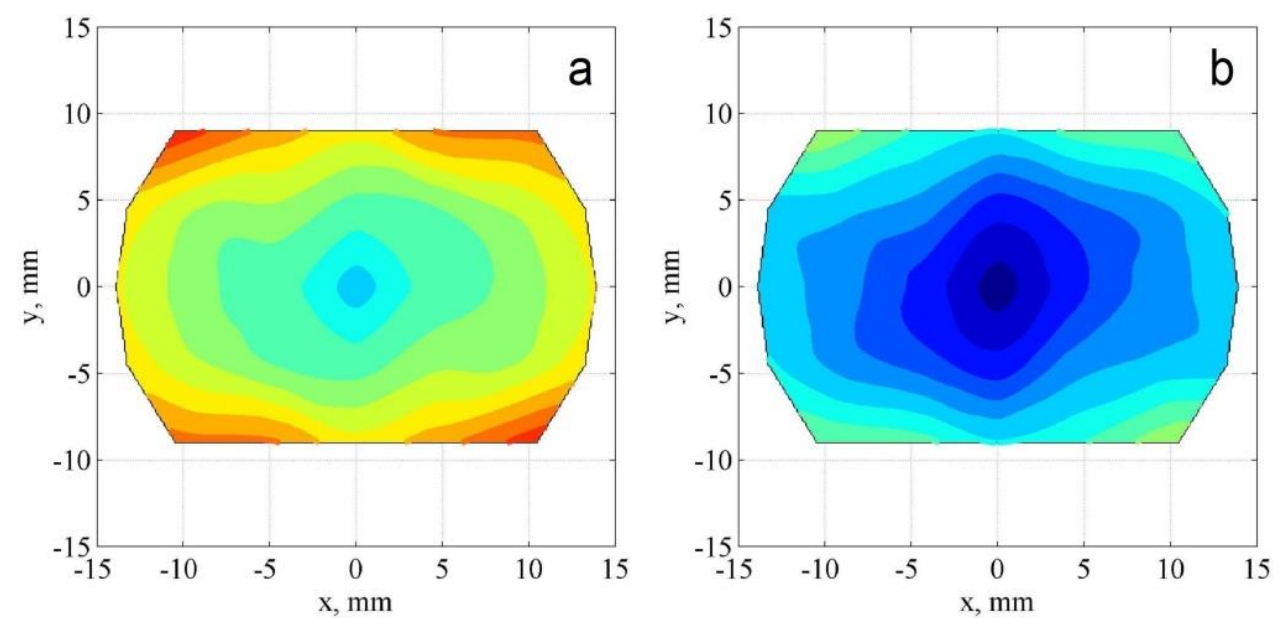

[MPa]

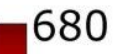

660

640

620

600
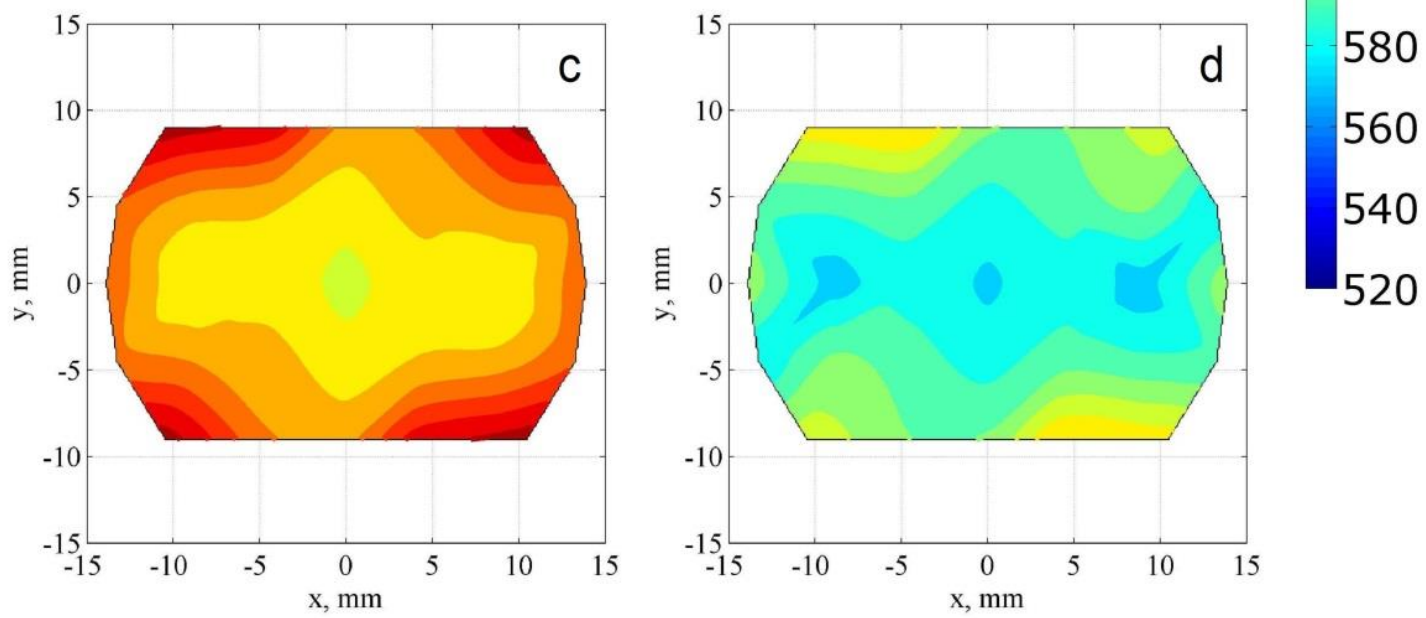

Fig. 5. Strength properties distribution over the billet cross-section: $(a, b)$ distribution of $\sigma_{U T S}$ and $\sigma_{Y S}$, respectively, for billet\#1 (2 passes at $350^{\circ} \mathrm{C}$ and $200 \mathrm{MPa}$ backpressure); (c,d) distribution of $\sigma_{U T S}$ and $\sigma_{Y S}$, respectively, for billet\#2 (4 passes at $350^{\circ} \mathrm{C}$ and $200 \mathrm{MPa}$ backpressure).

It can be seen from Figure 5 that, the values of strength properties are non-uniform within the cross-section of the billet after 2 passes, with lower values in the center and increasing 
monotonically towards the edge (see Figure 5a,b). These results are in accordance with the distribution of equivalent plastic strain within the cross-section of the billet shown in Figure 4. The non-uniformity of deformation results in inhomogeneous distribution of mechanical properties along the sample cross section.

At the same time, Figure 5c,d demonstrates a tendency towards decreasing non-uniformity of the strength characteristics along the billet cross-section with increasing numbers of TE passes. Figure 5c,d shows that after the forth TE pass the area with lowered strength properties shifted from the center of the billet towards the periphery of the cross-section. Table 1 summarizes results of tensile tests and shows data on variability of mechanical properties after 2 and 4 passes.

Table 1. Mean values of mechanical properties and their variability after 2 and 4 TE passes

\begin{tabular}{|c|c|c|c|c|c|c|c|c|}
\hline \multirow[b]{2}{*}{ Material condition: } & \multicolumn{2}{|c|}{$\sigma_{U T S}$} & \multicolumn{2}{|c|}{$\sigma_{Y S}$} & \multicolumn{2}{|c|}{$\delta$} & \multicolumn{2}{|c|}{$\psi$} \\
\hline & $\begin{array}{l}\text { mean* } \\
{[\mathrm{MPa}]} \\
{[\mathrm{a}]}\end{array}$ & $\begin{array}{l}C V^{* *} \\
{[\%]} \\
{[\mathrm{b}]}\end{array}$ & $\begin{array}{l}\text { mean } \\
{[\mathrm{MPa}]}\end{array}$ & $\begin{array}{l}C V \\
{[\%]}\end{array}$ & $\begin{array}{l}\text { mean } \\
{[\%]}\end{array}$ & $\begin{array}{l}C V \\
{[\%]}\end{array}$ & $\begin{array}{l}\text { mean } \\
{[\%]}\end{array}$ & $\begin{array}{l}C V \\
{[\%]}\end{array}$ \\
\hline As-received & 473 & 1.4 & 358 & 2.5 & 31 & 9.4 & 68 & 2.8 \\
\hline billet\#1 (2 passes) & 617 & 3.4 & 572 & 3.9 & 17.5 & 8.8 & 68 & 3.3 \\
\hline billet\#2 (4 passes) & 641 & 3.2 & 597 & 3.1 & 17.7 & 6.9 & 67 & 3.2 \\
\hline
\end{tabular}

[a] hereinafter mean is an average value of mechanical characteristic in the cross-section of the billet $[b]$ coefficient of variation $C V$ was calculated according to (2)

It can be seen from Table 1 that the $\sigma_{U T S}$ and $\sigma_{Y S}$ of the billet\#1 after 2 TE passes have increased by $30 \%$ and $60 \%$, respectively, compared to their initial values. The non-uniformity of the $\sigma_{U T S}$ and $\sigma_{Y S}$ distribution has increased after TE compared to the as-received condition; the $C V$ increased to $3.4 \%$ and $3.9 \%$, respectively. As a result, the $S D$ (standard deviation) of $\sigma_{U T S}$ and $\sigma_{Y S}$ slightly increased to the values $18-22 \mathrm{MPa}$. The $C V$ of plastic properties $\delta$ and $\psi$ remained nearly the same.

Following two TE cycles did not result in considerable increasing $\sigma_{U T S}$ and $\sigma_{Y S}$ (see billet\#2 in Table 1). However, a tendency towards increasing homogeneity of mechanical properties in 
the billet cross-section was observed. The $C V$ for all mechanical characteristics decreased slightly after the fourth pass. This observation suggests that, despite non-uniformity of deformation during TE, the distribution of mechanical properties along the sample crosssection becomes more homogenous with increasing numbers of TE passes.

Table 1 also demonstrates that the values of $\psi$ (reduction in area) remain at the level of asreceived material. This suggests that material processed by multiple TE passes maintain technological plasticity and can undergo further deformation by conventional metal forming techniques to obtain final shape of the product. At the same time, the value of $\delta$ (elongation to failure) decreased after TE processing (see Table 1). The $\delta$ is composed of uniform elongation before necking $\delta_{u}$ and reduction in area $\psi$. As mentioned above, the latter plastic property remained the same, so it can be assumed that decreasing of the $\delta$ was caused by decreasing of the $\delta_{u}$ value. This fact indicates high hardening of the material after TE processing. Taking into account decreased gap between $\sigma_{U T S}$ and $\sigma_{Y S}$ values after TE compared with as-received state, it can be concluded that hardening of the material is close to the upper limit values. A more detailed study on the ductility in UFG materials is given in an earlier study. ${ }^{[30]}$

\section{Discussion}

Figure 6 summarizes all the tensile test results plotted as mechanical properties versus accumulated plastic strain coordinate representation. These curves were constructed by combining the tensile test results, performed on small-size specimens cut out of the billets after 2 and 4 passes, with the maps of strain distribution within the billet cross-section obtained by FEA of multipass TE (see Figure 4). Each point in Figure 6 corresponds to a particular small-size specimen with a certain value of accumulated plastic strain. 

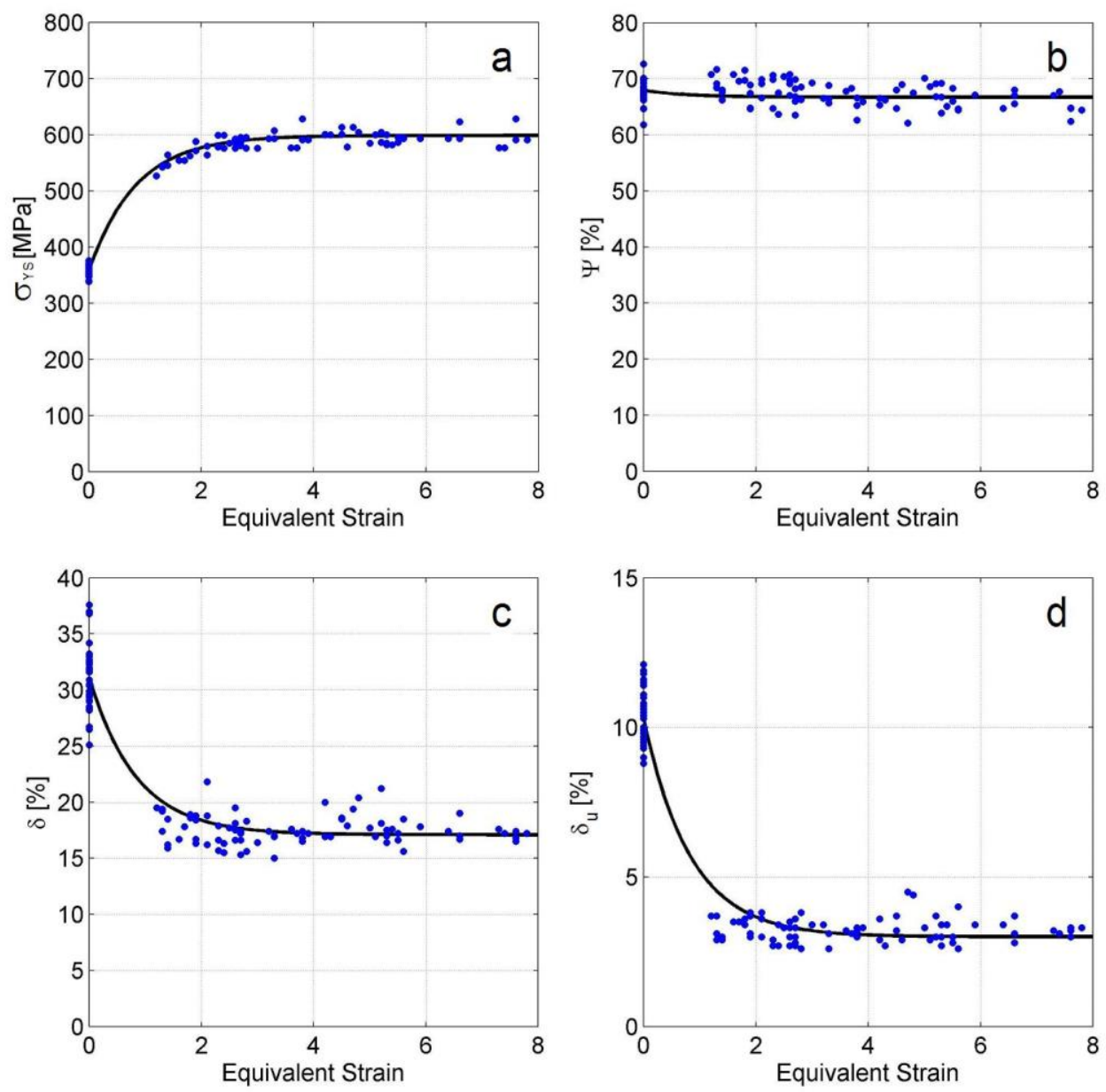

Fig. 6. Mechanical properties versus accumulated plastic strain plots: (a) yield stress $\sigma_{Y S}$, $M P a ;(b)$ reduction in area $\psi, \%$; (c) total elongation to failure $\delta, \% ;(d)$ uniform elongation $\delta_{u}, \%$.

The black trend line in Figure 6a was plotted by approximation of experimental data with the Equation (16)

$\sigma=\sigma_{S}-\left(\sigma_{S}-\sigma_{Y S}\right) \cdot \exp [-N \cdot e]$

where $\sigma_{S}$ is the steady state flow stress (599.1 MPa), $\sigma_{Y S}$ is the initial average yield stress (358.3 MPa), $N$ is the non-dimensional material constant (1.16).

The trend lines for Figure $6 \mathrm{~b}, \mathrm{c}, \mathrm{d}$ were plotted in a similar manner. The plots shown in Figure 6 demonstrate that the strength and plastic characteristics of $\mathrm{CP}$ titanium billets after TE attain a saturation level with the rise in accumulated plastic strain, i.e. the processed 
material demonstrates neither strain-hardening nor fracture. This fact indicates the occurrence of an ideal plastic state. It is reasonable to assume that its onset is caused by the stabilization of microstructure in the processed material. The saturation of properties occurs once the accumulated plastic strain exceeds some saturation level $e_{s}$. This saturation effect was obtained for CP Ti billets processed by TE under the following conditions:

- pressure in the deformation zone in the range 300 to $700 \mathrm{MPa}$;

- equivalent plastic strain in the range 4 to 8 ;

- and temperature range $300^{\circ} \mathrm{C}-400^{\circ} \mathrm{C}$.

For the case of $\mathrm{CP}$ Ti processed under the above mentioned conditions, the strain saturation level was $e_{s} \approx 4$. It can be seen from Figure 6 that CP titanium VT1-0 demonstrates ideal plastic flow in the equivalent strain range $\sim 4$ to 8 . With that the average value of $\sigma_{Y S}$ along the billet cross-section after 4 passes increased by $67 \%$ compared to the value for the initial state, i.e. from $\sigma_{Y S} \approx 360 \mathrm{MPa}$ to $\sigma_{Y S} \approx 600 \mathrm{MPa}$ (see Table 1 ). The maximum flow stress values were obtained for specimens sourced from the corners of the billet cross-section. The $\sigma_{Y S}$ reaches the values of $\sim 640 \mathrm{MPa}$ in these areas. It is important to also note that the main strength gain (95\% of the total strengthening after 4 passes) was obtained upon reaching an equivalent strain of $e \approx 2.5$. With a further increase in strain, the mechanical properties start to attain their saturation level. In addition, the $\delta$ and $\psi$ remain constant at $\sim 18 \%$ and $\sim 67 \%$, respectively, once the saturation strain level $\left(e_{s} \approx 4\right)$ is reached. With an increase in the number of passes, the zone where strain exceeds the saturation threshold gradually fills up the entire cross-section. This leads to leveling out of the mechanical properties and microstructure along the billet cross-section. ${ }^{[14,31]}$

On the basis of the observations above, as well as the fact that the equivalent strain in the center of the billet cross-section after one pass is $\sim 0.5$ (see Figure 4), one can assume that the mechanical properties in the axial area will reach those in the corners of billet after 8 passes, i.e. once the central region accumulates a strain of $e_{s} \approx 4$. 
Such saturation of strength properties and stabilization of microstructure are quite common and typically occur in any SPD technique with a simple shear deformation scheme, for instance, HPT. Such effect is recorded in a wide range of materials in ref. ${ }^{[18,19,20,22,32,33,34]}$ The possible explanation of mechanisms responsible for this phenomenon are described elsewhere. ${ }^{[33,35,36,37]}$

Figure 7 shows the calculated $\sigma_{Y S}$ values plotted as a function of the distance to the center $R$. $\sigma_{Y S}$ data in Figure 7 was calculated using the relation (16). It can be seen that the $\sigma_{Y S}$ has a high spread of values across the billet cross-section after the first pass. However, $\sigma_{Y S}$ value distribution becomes almost uniform already after the fourth pass. Data plotted in Figure 7 also confirm the above considerations regarding hardening of the central part of the billet cross-section (see the $\sigma_{Y S}$ values at $R=0$ ). It follows from Figure 7 that the axial zone of the billet demonstrates considerable strain hardening already after the second pass, while after the fourth pass, the $\sigma_{Y S}$ of the material in the center of the cross-section is already close to its maximum value.

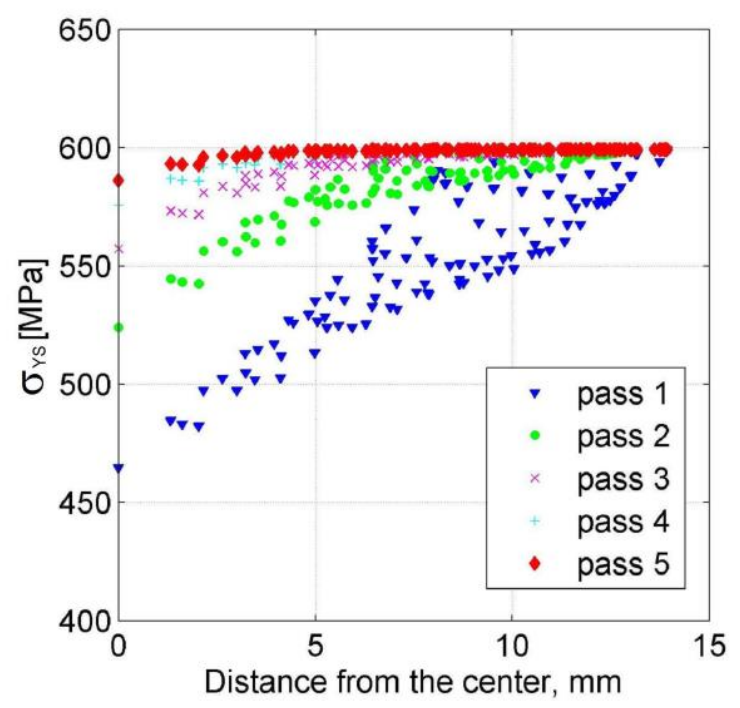

Fig. 7. The values of yield stress $\sigma_{Y S}$ calculated after various number of passes and plotted as a function of radius $R$ (distance to the center). 
In contrast to TE, similar plots for HPT process always start from the $\sigma_{Y S}$ of the initial material regardless of the number of revolutions, as the center of the material remains almost undeformed. This point corresponds to the initial state of the material at $R=0$ (see, for example, Figure 6 in ref. ${ }^{[18]}$ and Figure $3 a$ in ref. $\left.{ }^{[21]}\right)$. It is assumed that the principal distinction in strain hardening of the billet's axial zone during HPT and TE is caused by the difference in the deformation mode described in Section 3.1.

There are two distinctive features of the saturation effect in TE compared with that in HPT process. The first one is the fact that the saturation level of the strength properties in TE is lower than that in HPT process ${ }^{[38,39]}$ (particular values are discussed below). Another feature which distinguishes TE from HPT is the value of the accumulated strain, $e_{s}$, above which the material does not demonstrate strain-hardening anymore. This peculiarity lies in the fact that the strain-hardening limit in TE occurs at much smaller strain values compared with those in HPT. It follows from the analysis of the plots in Figure 6 that $e_{s} \approx 4$ for CP Ti processed by TE under the experimental conditions applied. In comparison, as observed in ref., ${ }^{[17]}$ the microhardness of Grade 2 CP titanium processed by HPT under a pressure of 3.0 GPa reached a saturation value of $\sim 305 \mathrm{Hv}$ after a shear strain of $\gamma \approx 150$, which corresponds to the equivalent von Mises strain of $e_{s} \approx 87$ (recalculated as $e=\frac{\gamma}{\sqrt{3}}$ ). The microhardness values for Ti samples processed by HPT under a higher pressure of $6.0 \mathrm{GPa}$ reached a saturation value of $\sim 320 \mathrm{Hv}$ after a lower shear strain of $\gamma \approx 50,{ }^{[17]}$ which corresponds to an equivalent von Mises strain of $e_{s} \approx 29$. Thus, although $e_{s}$ value in HPT differs essentially depending on the process parameters, it is at least 5 times higher than that observed for CP Ti processed by TE. It should be noted that there could be two possible reasons causing the peculiarities of the saturation effect in TE compared with HPT process. Firstly, it could be the cyclic pattern of deformation during TE. Furthermore, TE process provides significantly lower hydrostatic pressure in the deformation zone compared with HPT. The presence of much higher 
hydrostatic pressure during HPT leads to the later onset of grain refinement and hardening saturation limits. It also results in smaller final grain size and higher strength properties of the processed material compared with the ones processed by TE. For example, it was shown in ref. $^{[38]}$ that HPT processing of CP Ti Grade 4 under a pressure of 6 GPa leads to strong grain refinement from $40 \mu \mathrm{m}$ to $105-120 \mathrm{~nm}$ and an enhancement of the $\sigma_{U T S}$ from 700 to 1600 MPa. Another study ${ }^{[39]}$ carried out on CP Ti VT1-0 (the same grade of CP Ti as was used in this study) has shown that after HPT under an applied pressure of $5 \mathrm{GPa}$, the material demonstrates $\sigma_{Y S}$ values higher than $800 \mathrm{MPa}, \sigma_{U T S}$ of $980 \mathrm{MPa}$ and elongation to fracture of 12\%. These strength properties are much higher than those obtained after TE. Such high levels of strength and grain refinement cannot be achieved by TE or any other SPD process with much lower (compared with HPT) hydrostatic pressure in the deformation zone.

\section{Conclusions}

1. During TE processing, the strength and plastic characteristics of $\mathrm{CP}$ titanium attain saturation when the following conditions are met:

- pressure in the deformation zone in the range 300-700 MPa;

- equivalent plastic strain in the range 4-8;

- temperature in the range $300^{\circ} \mathrm{C}-400^{\circ} \mathrm{C}$.

Under these conditions, the material demonstrates neither strain-hardening nor fracture. This fact indicates the occurrence of an ideal plastic state in the processed material.

2. It was theoretically and experimentally proved that the strain-hardening of material on the extrusion axis during TE reaches its saturation value much earlier than that observed during HPT process. The assumption behind this observation is that the time vs strain count-down starts from the moment when an ideal plastic state is reached on the periphery of the billet. It was shown that the values of mechanical properties in the center of the titanium billet become 
exactly equal to those in the peripheral areas already after $8 \mathrm{TE}$ passes, when the accumulated plastic strain on the axis reaches the saturation level $e_{s} \approx 4$.

3. The yield stress value of titanium billet processed by TE in the ideal plastic state is lower than those obtained during HPT processing, i.e. the ideal plastic state requires much smaller values of accumulated strain and, consequently, starts earlier.

4. The above listed peculiarities of TE, compared with HPT, are caused by the different symmetry of these two processes, as well as the big difference in hydrostatic pressure in the deformation zone. It is shown that the central part of the billet is deformed during TE due to asymmetrical cross-section of the twist die channel.

Received: ((will be filled in by the editorial staff))

Revised: ((will be filled in by the editorial staff))

Published online: ((will be filled in by the editorial staff))

[1] R.Z. Valiev, Y. Estrin, Z. Horita, T.G. Langdon, M.J. Zehetbauer, Y.T. Zhu, JOM

2006, 58,33 .

[2] Y. Estrin, A. Vinogradov, Acta Mater. 2013, 61, 782.

[3] P.W. Bridgman, J. Appl. Phys. 1937, 8, 328.

[4] P.W. Bridgman, J. Appl. Phys. 1943, 14, 273.

[5] A.P. Zhilyaev, T.G. Langdon, Prog. Mater. Sci. 2008, 53, 893.

[6] R.K. Islamgaliev, N.F. Yunusova, I.N. Sabirov, A.V. Sergueeva, R.Z. Valiev, Mater.

Sci. Eng. A 2001, 319, 877.

[7] G. Sakai, K. Nakamura, Z. Horita, T.G. Langdon, Mater. Sci. Eng. A 2005, 406, 268.

[8] Z. Horita, T.G. Langdon, Scr. Mater. 2008, 58, 1029.

[9] K. Edalati, Z. Horita, J. Mater. Sci. 2010, 45, 4578.

[10] V. Fedorov, Yu. Ivanisenko, B. Baretzky, H. Hahn, Patent DE 102013213 072.4, 2013. 
[11] Y.Y. Beygelzimer, V.N. Varyukhin, S.G. Synkov, A.N. Sapronov, V.G. Synkov, Phys. Technol. High Pressures 1999, 9, 109.

[12] Y. Beygelzimer, D. Orlov, V. Varyukhin, in TMS Annual Meeting and Exhibition

(Eds: Y. T. Zhu, T. G. Langdon, R. S. Mishra, S. L. Semiatin, M. J. Saran, T. C. Lowe), TMS, Seattle, Washington, USA 2002, p. 297.

[13] S.R. Bahadori, S.A.A.A. Mousavi, Mater. Sci. Eng. A 2011, 528, 6527.

[14] Y. Beygelzimer, D. Orlov, A. Korshunov, S. Synkov, V. Varyukhin, I. Vedernikova, A. Reshetov, A. Synkov, L. Polyakov, I. Korotchenkova, Solid State Phenom. 2006, 114, 69.

[15] Y. Beygelzimer, A. Reshetov, S. Synkov, O. Prokof'eva, R. Kulagin, J. Mater.

Process. Technol. 2009, 209, 3650.

[16] R.Z. Valiev, A.P. Zhilyaev, T.G. Langdon, Bulk Nanostructured Materials:

Fundamentals and Applications, John Willey\&Sons, Hoboken New Jersey, USA 2013.

[17] C.T. Wang, A.G. Fox, T.G. Langdon, J. Mater. Sci. 2014, 49, 6558.

[18] A. Vorhauer, R. Pippan, Scr. Mater. 2004, 51, 921.

[19] Y.Z. Tian, J. Freudenberger, R. Pippan, Z.F. Zhang, Mater. Sci. Eng. A 2013, 568, 184.

[20] M. Kawasaki, J. Foissey, T.G. Langdon, Mater. Sci. Eng. A 2013, 561, 118.

[21] Y.P. Song, M.M. Chen, B.Y. Xu, D.S. Gao, H.S. Kim, Mater. Sci. Appl. 2016, 7, 673.

[22] M. Kawasaki, R.B. Figueiredo, T.G. Langdon, Acta Mater. 2011, 59, 308.

[23] X.H. An, S.D. Wu, Z.F. Zhang, R.B. Figueiredo, N. Gao, T.G. Langdon, Scr. Mater. 2010, 63, 560 .

[24] A. Bachmaier, J. Keckes, K.S. Kormout, R. Pippan, Philos. Mag. Lett. 2014, 94, 9.

[25] A.I. Korshunov, I.I. Vedernikova, L.V. Polyakov, T.N. Kravchenko, A.A. Smolyakov, V.P. Solovyov, Mater. Sci. Forum 2005, 503-504, 693.

[26] A.I. Korshunov, I.I. Vedernikova, L.V. Polyakov, T.N. Kravchenko, A.A. Smolyakov, V.P. Soloviev, Rev. Adv. Mater. Sci. 2005, 10, 235. 
[27] M.I. Latypov, M.-G. Lee, Y. Beygelzimer, R. Kulagin, H.S. Kim, Met. Mater. Int. 2015, 21, 569 .

[28] R. Kulagin, M. Latypov, H.S. Kim, V. Varyukhin, Y. Beygelzimer, Metall. Mater. Trans. A 2013, 44, 3211.

[29] Y. Beygelzimer, R. Kulagin, Y. Estrin, L.S. Toth, H.S. Kim, M.I. Latypov, Adv. Eng. Mater. 2017, 19, DOI 10.1002/adem.201600873.

[30] Y. Beygelzimer, O. Prokof'eva, R. Kulagin, V. Varyukhin, S. Synkov, Mater. Sci. Forum 2010, 633-634, 223.

[31] A. Reshetov, A. Korshunov, A. Smolyakov, Y.Beygelzimer, V. Varyukhin,

I. Kaganova, A. Morozov, Mater. Sci. Forum 2011, 667-669, 851.

[32] P.W. Bridgman, Studies in Large Plastic Flow and Fracture, with Special Emphasis on the Effects of Hydrostatic Pressure, Harvard University Press, Cambridge, USA 1952.

[33] R. Pippan, F. Wetscher, M. Hafok, A. Vorhauer, I. Sabirov, Adv. Eng. Mater. 2006, 8, 1046.

[34] A. Bachmaier, M. Hafok, R. Schuster, R. Pippan, Rev. Adv. Mater. Sci. 2010, 25, 16.

[35] H.W. Zhang, X. Huang, N. Hansen, Acta Mater. 2008, 56, 5451.

[36] R. Pippan, S. Scheriau, A. Taylor, M. Hafok, A. Hohenwarter, A. Bachmaier, Annu. Rev. Mater. Res. 2010, 40, 319.

[37] Y. Beygelzimer, N. Lavrinenko, arXiv:1206.5055 [cond-mat.mtrl-sci] 2012.

[38] R.K. Islamgaliev, V.U. Kazyhanov, L.O. Shestakova, A.V. Sharafutdinov, R.Z. Valiev, Mater. Sci. Eng. A 2008, 493, 190.

[39] R.Z. Valiev, A.V. Sergueeva, A.K. Mukherjee, Scr. Mater. 2003, 49, 669. 
This paper deals with the analysis of strength and plastic characteristics of commercially pure titanium specimens as a function of equivalent plastic strain accumulated during Twist Extrusion (TE) process. It is shown experimentally that multipass TE leads to the saturation of mechanical properties in the billet. This fact indicates the occurrence of an ideal plastic state. The metal flow during TE and High Pressure Torsion processes is compared. An explanation for the hardening central zone of the billet during TE is offered. The differences in final mechanical properties of titanium billets processed by these two techniques are studied and explained.

\section{A. Reshetov, * R. Kulagin, A. Korshunov, and Y. Beygelzimer}

The Occurrence of Ideal Plastic State in CP Titanium Processed by Twist Extrusion

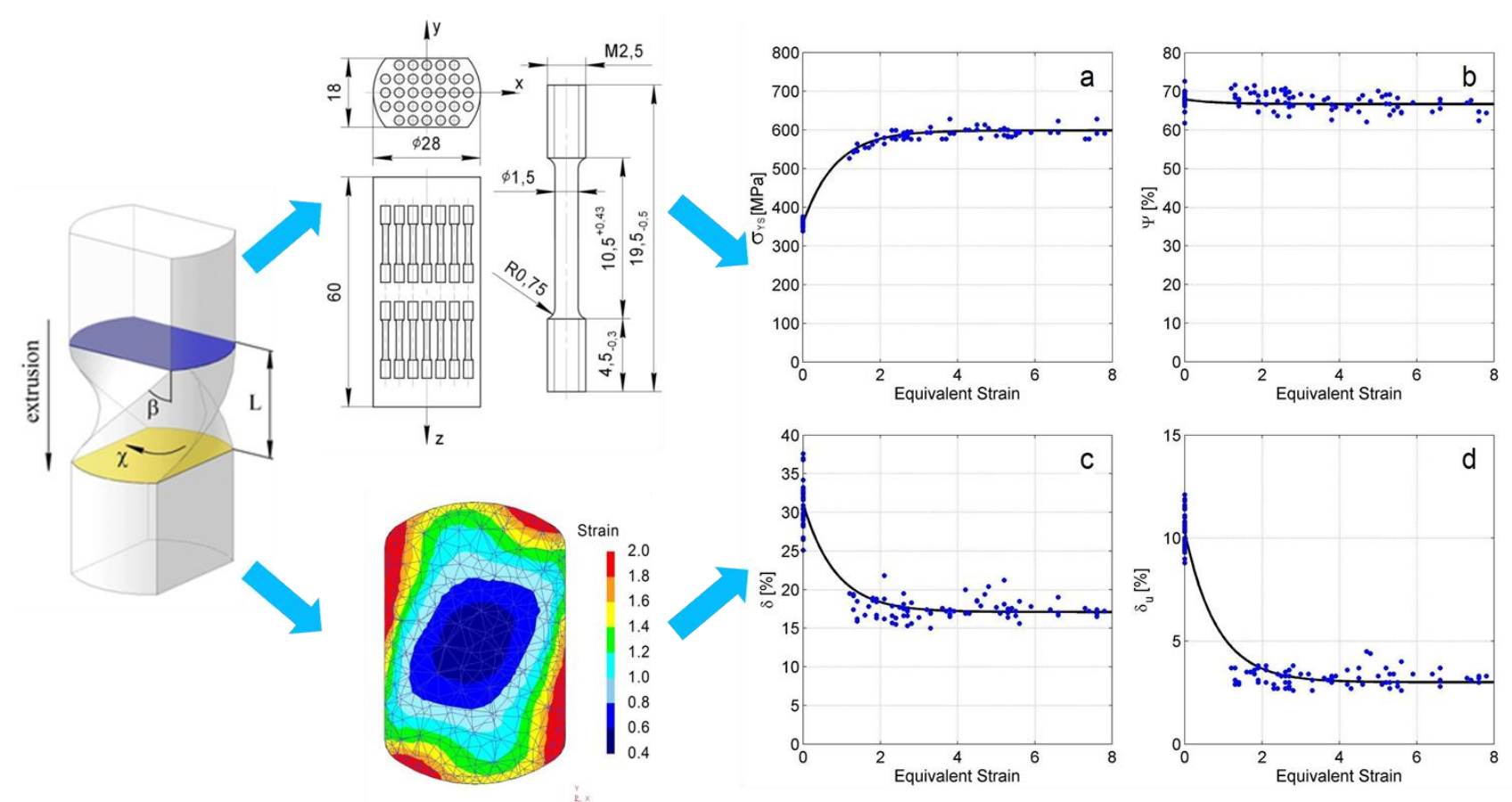

